\title{
An experimental study of the molecular mixing process in an axisymmetric laminar vortex ring
}

\author{
Kenneth B. Southerland, John R. Porter III, Werner J. A. Dahm, and Kenneth A. Buch \\ Department of Aerospace Engineering, The University of Michigan, Ann Arbor, Michigan 48109-2140
}

(Received 18 September 1990; accepted 21 December 1990)

\begin{abstract}
Results are presented from an experimental study of the molecular mixing of a dynamically passive conserved scalar quantity in an axisymmetric laminar vortex ring. The experiments are based on highly resolved laser-induced fluorescence imaging measurements of the scalar field $\zeta(x, t)$ in the diametral plane of the ring, from which the evolution of the molecular mixing rate field $\nabla \zeta \cdot \nabla \zeta(\mathrm{x}, t)$ can be directly examined. In particular, the structure and dynamics of the mixing process arc addressed during the three characteristic stages in the ring evolution, namely, (i) the ring generation stage, (ii) the ring pinch-off stage, and (iii) the asymptotic stage of the ring. Results show a layering of the mixing process in which the diffusional cancellation term $\nabla(\nabla \zeta): \nabla(\nabla \zeta)$ plays a major role in setting the overall mixing rate achieved. The scalar field measurements are also used to extract detailed information about the underlying velocity field in the ring.
\end{abstract}

\section{INTRODUCTION}

A stable laminar vortex ring is formed when an impulse is imparted on a fluid in an axisymmetric manner. The dynamics of the resulting vorticity field during various stages of the evolution of such vortex rings has been a subject of considerable interest for over 50 years. ${ }^{1-17}$ Indeed the laminar vortex ring is one of the most fundamental of the canonical flow fields used in the study of vorticity dynamics. This interest comes largely from the fact that the simple structure of the ring lends itself well to both experimental and theoretical investigation. These earlier studies have shown that many aspects of the vortex ring formation and propagation processes differ markedly from their classical descriptions based on various potential flow models.

In this same sense, the laminar vortex ring provides a relatively simple flow field, simultaneously accessible to experimental, numerical, and theoretical study, in which understanding of the physics of the molecular mixing process in fluid tlows also can be meaningfully examined and extended. Yet the mixing processes within such a laminar vortex ring do not appear to have bcen documented in any significant detail. There are of course several problems in which these mixing processes in the ring are of direct relevance, and many others for which they provide a simple canonical representation of potentially more complicated processes. Examples range from the mixing processes in a rising atmospheric thermal and their implications for cloud formation, to the processes occurring during the interaction of a vortex with a premixed flame ${ }^{8}$ as a simple representation of the interaction of a turbulent flow with a flame front. In a broader sense, however, the laminar vortex ring provides a relatively simple and carefully controllable flow field in which the.interaction of strain and diffusion on the mixing process can in principle be studied in considerable detail.

The study described here concerns itself with the mixing of dynamically passive conserved scalar quantities in such an axisymmetric laminar vortex ring. By this we refer to quantities that are advected by the fluid and can diffuse relative to the fluid, but which are neither created nor destroyed in the flow and which have no direct influence on the underlying flow field. We focus here on presenting a relatively detailed documentation based on experimental measurements of various features of the molecular mixing of such scalars in the ring, and on their interpretation in terms of the dynamics of the associated mixing rate field. The presentation is organized as follows. In Sec. II we give a brief summary of the formulation adopted here to quantify the molecular mixing process. In Sec. III we describe the experimental technique used to generate the rings and to measure the mixing rate field within them. Results are presented in Sec. IV, where we discuss the structure and evolution of the mixing rate field during the ring formation phase, the pinch-off phase, and the asymptotic phase. The extraction of the velocity field from such measurements of the conserved scalar field is discussed in Sec. V and in Sec. VI we make several concluding remarks concerning the mixing processes within the ring.

\section{MIXING RATE FORMULATION}

The mixing process within the ring can be formulated in terms of a dynamically passive conserved scalar field $\zeta(\mathbf{x}, t)$, represented here by the concentration of an inert dye carried by one of the fluids in the ring. In such a conserved scalar field, the local scalar value can change only through advection of the scalar with the fluid and molecular diffusion of the scalar relative to the fluid, and thus satisfies the advection-diffusion equation

$$
\left(\frac{\partial}{\partial t}+\mathbf{u} \cdot \boldsymbol{\nabla}-\frac{1}{\operatorname{ReSc}} \nabla^{2}\right) \xi(\mathbf{x}, t)=0 .
$$

Here all variables have been made nondimensional by normalization with suitable reference length and velocity scales. From Eq. (1) the scalar energy per unit mass $\frac{1}{2} \zeta^{2}$, defined 
analogous to the kinetic energy per unit mass $\frac{1}{2} u^{2}$, where $u \equiv|\mathbf{u}|$, follows the transport equation,

$$
\left(\frac{\partial}{\partial t}+u \cdot \nabla-\frac{1}{\operatorname{ReSc}} \nabla^{2}\right) \frac{1}{2} \zeta^{2}(\mathbf{x}, t)=-\frac{1}{\operatorname{ReSc}} \nabla \zeta \cdot \nabla \zeta,
$$

where the same conservative advection-diffusion operator appears on the left. The term on the right then gives the scalar energy dissipation rate per unit mass, namely the local instantaneous rate at which nonuniformities in the scalar energy field are being reduced by molecular diffusion of the scalar in the flow. The scalar dissipation field $\nabla \xi \cdot \nabla \xi(\mathbf{x}, t)$ is therefore often taken to define the instantaneous molecular mixing rate field in the flow. We note that the molecular mixing rate is sometimes alternately chosen as the magnitude of the scalar gradient, $|\nabla \xi(\mathrm{x}, t)|$, though the logarithm of these two definitions differs only by a constant factor. Most of the results presented here are in terms of the logarithm of the mixing rate, and thus apply equally to both definitions.

From Eq. (1), the scalar dissipation field in Eq. (2) can be seen to follow the transport equation

$$
\begin{aligned}
& \left(\frac{\partial}{\partial t}+\mathbf{u} \cdot \nabla-\frac{1}{\operatorname{Re~Sc}} \nabla^{2}\right) \frac{1}{2}(\nabla \zeta \cdot \nabla \zeta) \\
& =-(\nabla \zeta \cdot \bullet \cdot \nabla \zeta)-\frac{1}{\operatorname{ReSc}} \nabla(\nabla \zeta): \nabla(\nabla \zeta),
\end{aligned}
$$

where the same conservative advection-diffusion operator is on the left. In the first term on the right, $\epsilon \equiv \frac{1}{2}\left(\nabla \mathbf{u}+\nabla \mathbf{u}^{T}\right)$ is the local strain rate field, and the symmetric contraction simply selects the normal strain rate along the scalar gradient vector direction as $-\epsilon_{\nabla \xi \nabla \xi}(\nabla \xi \cdot \nabla \xi)$. The remaining components of the strain rate act only to change the gradient vector orientation, but do not affect its magnitude. In this form, it can be readily seen that this term simply accounts for the reduction in scalar gradient magnitude due to extensional straining along the gradient direction, or conversely the increase in dissipation that results when the scalar gradient is compressed. The second term on the right in Eq. (3) is strictly negative, and gives the reduction in dissipation due to diffusional cancellation of gradients in the scalar dissipation field. It will be seen later that this term plays a key role in determining the evolution of the mixing rate field at various stages in the ring.

\section{EXPERIMENTAL TECHNIQUE}

The experimental technique used to generate the axisymmetric laminar vortex rings in this investigation is described elsewhere ${ }^{18}$ and will be only briefly summarized here. Each ring was formed by discharging a volume of fluid through a round nozzle to form a thin cylindrical sheet of vorticity. This vortex sheet rolls up and develops into a stable ring that propagates away from the nozzle. The discharged fluid consisted of a uniform, weak, aqueous solution of a laser fluorescent dye (disodium fluorescein) that was pneumatically driven through a contoured axisymmetric nozzle (shown in Fig. 1) by driving its free surface in a plenum with a high-pressure air stream metered through a micrometercontrolled, variable throat orifice held at sonic conditions

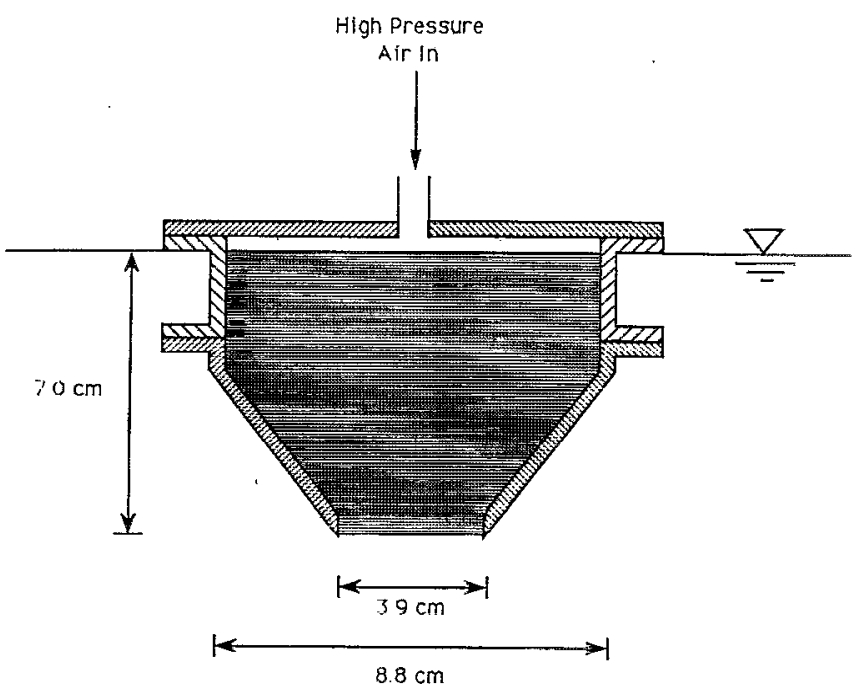

FIG. 1. Axisymmetric nozzle geometry used to generate the laminar vortex rings in this study.

with a constant upstream pressure. A solenoid valve in this high-pressure air line was opened and closed via a variable delay timing circuit to discharge the dye-containing plenum fluid. For a given upstream pressure, the area of the sonic metering orifice set the air flowrate into the plenum, and the solenoid delay time (typically $50-100 \mathrm{msec}$ ) set the total amount of air supplied to the plcnum. To keep the otherwise impulsive pressure rise driving the plenum fluid from exciting oscillations at the natural frequency of the second-order system formed by the compressibility of the air and the fluid mass in the plenum, the pressure rise was low-pass filtered by placing an adjustable volume of air in parallel with the plenum. This pneumatic drive arrangement allowed for a very low disturbance level and a high degree of symmetry in the vortex sheet generation and roll-up processes that form the ring. The nozzle had an exit diameter of $3.9 \mathrm{~cm}$ and produced rings with a center-to-center diameter $a \approx 4.9 \mathrm{~cm}$ under all operating conditions. The ring circulation $\Gamma$ was estimated from the integral of the exit velocity over time, and the corresponding Reynolds numbers $\operatorname{Re} \equiv(\Gamma / v)$ were typically in the range from 5000 to 10000 . The Schmidt number $\mathrm{Sc} \equiv(v / D)$ for the dye solution in water is $O\left(10^{3}\right)$, giving $\operatorname{Re} \mathrm{Sc} \approx O\left(10^{7}\right)$ in Eqs. (1) $-(3)$.

We measured the evolution of the resulting axisymmetric scalar field $\zeta(\mathbf{x}, t)$ by quantitative imaging of laser-induced fluorescence from dye-containing fluid along the path of a thin laser beam swept through the diametral plane of the ring. The imaging and data acquisition systems used for this are also described in detail elsewhere ${ }^{19,20}$ and will be only briefly summarized here. The measurements were based on high-speed acquisition of 256 successive $256 \times 256$ spatial data planes of the fluorescence intensity from the dye containing fluid carried by the ring. The fluorescence was projected onto a planar imaging array scanned at a pixel rate of $8.60 \mathrm{MHz}$, giving a net imaging rate of 100 planes $/ \mathrm{sec}$. The output from the array was serially acquired through a programmable digital port interface slaved to the array clock, 
digitized to 8-bit digital resolution, and ported directly into $16 \mathrm{MB}$ of high-speed image memory. A galvanometric mirror scanner was slaved to the timing signals driving the array to synchronize the laser beam sweep with the data acquisition. The resulting measured fluorescence intensity is related to the scalar field $\zeta(\mathbf{x}, t)$ through a measured image transfer function and a simple attenuation integral. Although the attenuation integral is important for correctly obtaining the scalar field, the principal interest here is in the mixing rate field $\nabla \xi \cdot \nabla \xi(\mathbf{x}, t)$ for which the effect of the relatively weak attenuation was neglected. Because of the axisymmetry of the ring, the gradient vector $\nabla \zeta(\mathbf{x}, t)$ has only in-plane components and can be completely determincd from these measurements in the diametral plane. The gradient vector field was obtained here by direct differentiation of the measurements using linear central difference approximations without any explicit smoothing or filtering of the results.

\section{RESULTS}

The results from these measurements suggest that the evolution of the mixing process within such an axisymmetric laminar vortex ring can be usefully classified into three relatively distinct stages, namely (i) a ring generation stage, (ii) a "pinch-off" stage, and (iii) an asymptotic stage. In this section, we present measurements of the mixing rate field in each of these stages and describe their particular characteristics.

\section{A. Generation phase}

The ring generation phase extends from the moment at which the impulse is first applied until the beginning of the pinch off phase. During this stage, the evolution of the scalar field is dominated almost entirely by the roll-up of the thin cylindrical vortex sheet emanating from the nozzle. A typical sequence of images showing the resulting roll-up of the scalar field $\zeta(\mathbf{x}, t)$ is presented in Fig. 2, and the sequence showing the associated mixing rate field $\log \nabla \zeta \cdot \nabla \zeta(\mathbf{x}, t)$ is given in Fig. 3. The sequence begins shortly after the pressure rise was first applied to the plenum, and shows only every 12 th frame from the imaging data, corresponding to a time interval between successive panels shown of $\left(\Delta t \cdot \Gamma / 2 \pi a^{2}\right) \approx 0.054$. As the axes indicate, the orange border near the top of each frame in Fig. 2 corresponds to the exit plane of the nozzle, and the imaged region extends nearly to the centerline of the flow. Pseudocolor assignments are used to denote the scalar and scalar dissipation values in each of these sequences, with the highest $0.1 \%$ of the dissipation values corresponding to pure red in Fig. 3. Here $D_{0}$ is the nozzle exit diameter and $\xi_{0}$ denotes the scalar value at the nozzle exit plane. In the earliest frame presented here, the scalar field at the front of the ring shows the effects of some slight stirring that has occurred between the plenum and ambient fluids due to small residual motions in the tank before the ring was generated. In the subsequent frames, it can be seen that the large stretching of the dye interface
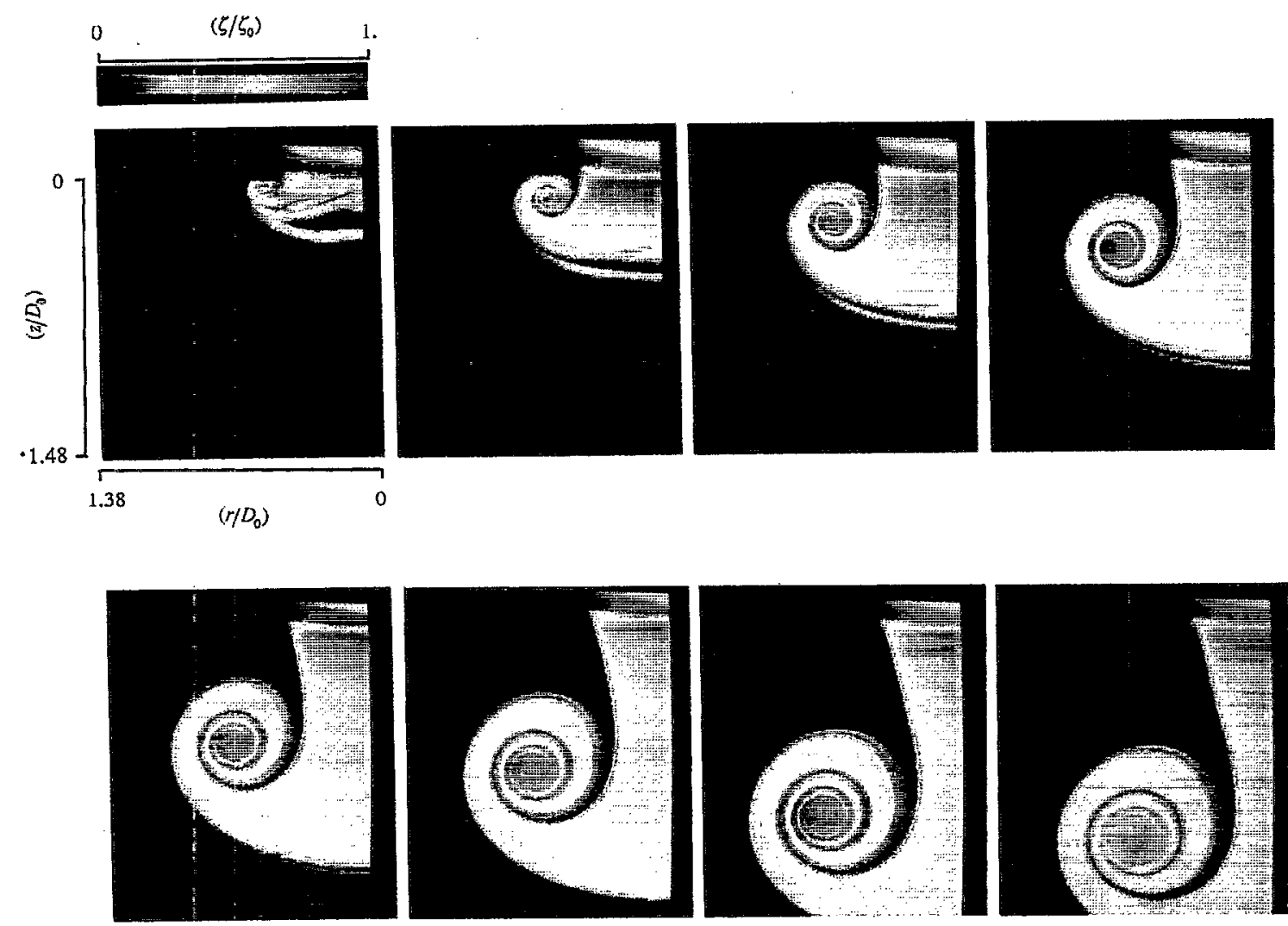

FIG. 2. Evolution of the scalar field $\zeta(x, t)$ during the ring formation phase. The dimensionless time between successive panels shown is $\left(\Delta t \cdot \Gamma / 2 \pi a^{2}\right) \approx 0.054$. 

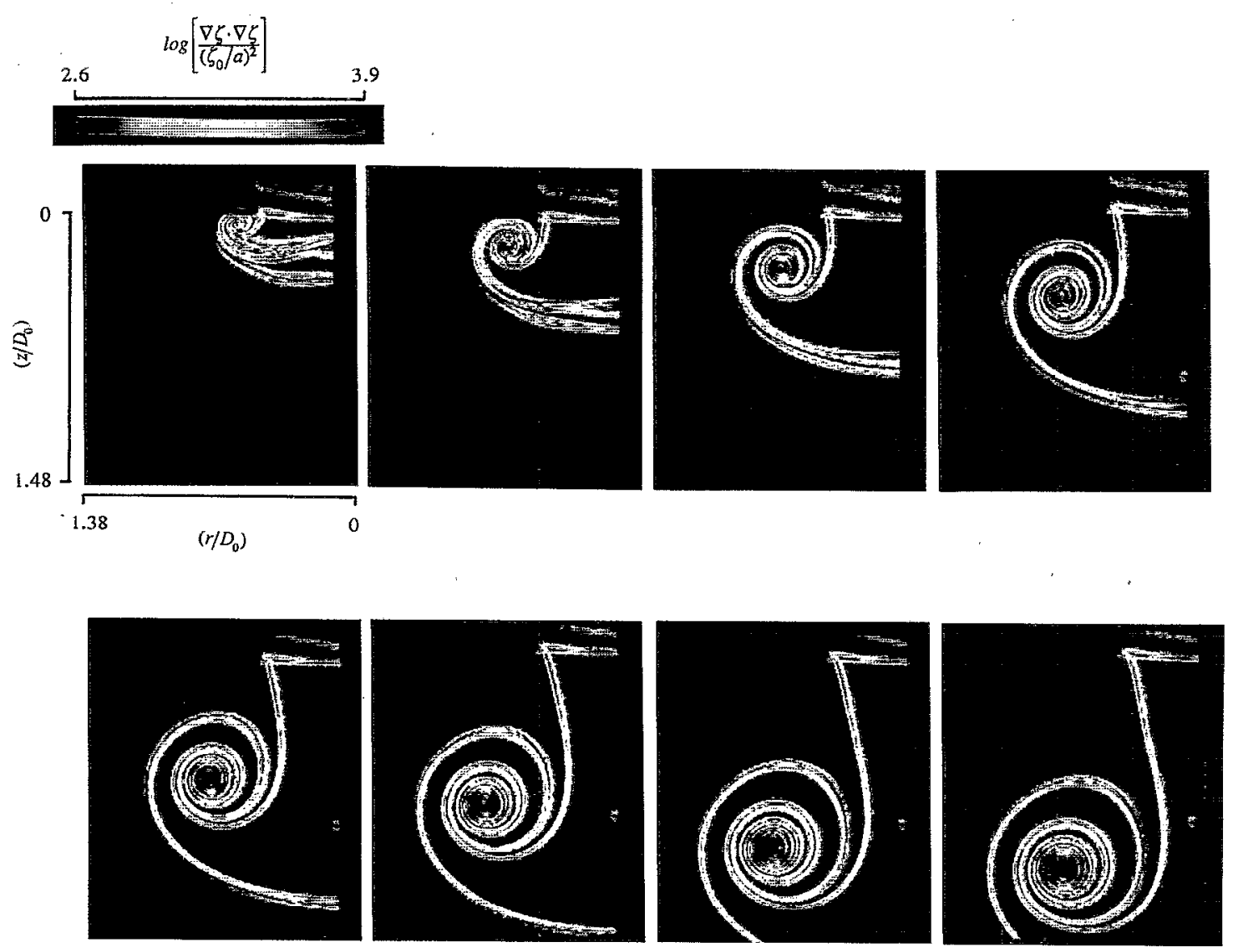

FIG. 3. The logarithm of the scaled mixing rate lield $\log \nabla \xi \cdot \nabla \zeta(\mathbf{x}, t)$ obtained from the scalar field shown in Fig. 2 .

extending from the forward stagnation point of the ring leads to a rapid compression of the structure normal to this interface, producing a relatively clean front in later frames.

Of particular interest here is the immediate rolling up into a tight spiral structure of the scalar interface in Fig. 2 and the associated spiral scalar dissipation layer in Fig. 3. These features can be seen to correspond to the sides of the otherwise cylindrical volume of dyed plenum fluid emanating from the nozzle. Indeed, there is evidence of a spiral roll up of this interface even in the earliest frames in which any motion of the fluid is discernible. The trajectory of the resulting vortex can also be seen here, though it must be cautioned that experiments by Auerbach ${ }^{1,2}$ as well as by Irdmussa and Garris ${ }^{5}$ indicate that the trajectory can depend quite strongly on the details of both the geometry and the process used to generate the ring. As a result, while most of the phenomena documented here appear to be characteristic of the mixing process in all laminar vortex rings, there are likely to be some comparatively minor aspects that are specific to the particular ring formation mechanism used here.

Overall the spiral roll-up of the scalar dissipation layer emanating from the nozzle lip in Fig. 3 is strongly suggestive of the expected form of the cylindrical vortex sheet that also must emanate from the nozzle. There is in fact an interesting similarity in the dynamics of the vorticity and scalar dissipation fields. In particular, note that Eq. (3) for the scalar dissipation $\nabla \xi \cdot \nabla \zeta$ is very similar to the transport equation for the square of the vorticity magnitude $\omega \cdot \omega$, sometimes referred to as the enstrophy, which takes the form

$$
\left(\frac{\partial}{\partial t}+\mathbf{u} \cdot \nabla-\frac{1}{\operatorname{Re}} \nabla^{2}\right) \frac{1}{2}(\omega \cdot \omega)=(\omega \cdot \mathbf{E} \cdot \omega)-\frac{1}{\operatorname{Re}} \nabla \omega: \nabla \omega .
$$

Comparison with Eq. (3) shows that the symmetric contraction in the first term on the right now selects the normal strain rate along the vorticity vector direction, namely $+\epsilon_{\omega \omega}(\omega \cdot \omega)$. The further difference in sign reflects the fact that extensional strain along the vorticity vector direction acts to increase its magnitude. Here, due to the axisymmetry of the ring, the scalar gradient and vorticity vectors will lie normal to each other, and thus the component of the strain rate that affects their evolution will be different. Interestingly though, in flows where $\nabla \xi$ and $\omega$ are aligned, the dynamics of the scalar dissipation at a given value of the dimensionless scalar diffusivity $\mathrm{Re} \mathrm{Sc}$ will be entirely parallel to those of the vorticity magnitude when Re is increased to this same value. Of course, in the vortex ring, the initial condition for the scalar dissipation includes a nonzero disk in the nozzle exit plane that has no parallel in the vorticity field.

We now turn to the effect of the diffusional cancellation term in Eq. (3) and examine its consequences when the dissipation layer extending from the forward stagnation point 
begins to interact with the layer emanating from the nozzle lip. This occurs along the spiral windings of the vortex as the core is approached. As was noted in Sec. II, this effect acts to reduce the rate of mixing in the vortex core. Thus even though an increasing amount of interfacial area between the plenum and ambient fluids is created by the roll-up of the vortex during this formation phase, the diffusional cancellation that occurs between adjacent arms of the spiral roll acts to limit the total mixing rate that is achieved. Another further issue that can be addressed from these results is the role that the formation process plays in determining the volume ratio of plenum and ambient fluids that will eventually be trapped in the Kelvin oval formed by the ring. The pinch off process that finally ends the further entrainment of plenum and ambient fluid into this oval is discussed in the following section. Here we can note, however, that the details of the spiral roll-up seen during the vortex formation stage in Figs. 2 and 3 clearly plays a major role in determining this ratio. In this manner, the geometry and the process used to generate the ring would be expected to play a significant role in setting the final ratio achieved by the ring, as discussed by Auerbach. ${ }^{2}$

\section{B. Pinch-off phase}

This stage is characterized by the "pinch-off" process shown in Fig. 4, where for brevity we present results for the mixing rate field $\log \nabla \xi \cdot \nabla \zeta(x, t)$ only. The sequence shown now consists of every 20 th frame from the imaged data, corresponding to a time interval between successive panels of $\left(\Delta t \cdot \Gamma / 2 \pi a^{2}\right) \approx 0.089$, and beginning at roughly the end of the ring formation stage described above. The quantitative color values are assigned as shown, with the highest $0.1 \%$ of the mixing rates again corresponding to pure red. The key feature in this phase is the diffusional cancellation between the two scalar dissipation layers seen in this diametral plane that intersects the initially cylindrical dissipation surface emanating from the nozzle. In the sequence in Fig. 4, this cancellation is most readily apparent between the two dissipation layers which at this time still connect the ring to the nozzle. At relatively early times these layers are still widely separated, but the strain field induced by the ring then begins to draw them together on a relatively fast time scale. A short time later, the two layers begin to interact strongly and, owing to the cancellation term in Eq. (3), the mixing rate rapidly drops to zero. Beyond this point, the ring is no longer "connected" to the nozzle and all of the plenum fluid that will eventually reside in the Kelvin oval has been committed to the ring. The ambient fluid trapped between the streamlines passing through the forward and rearward stagnation points then simply continues to wrap on an increasingly finer scale with this plenum fluid during the asymptotic stage.

Aside from the diffusional cancellation noted above, there is of course additional cancellation of this type that
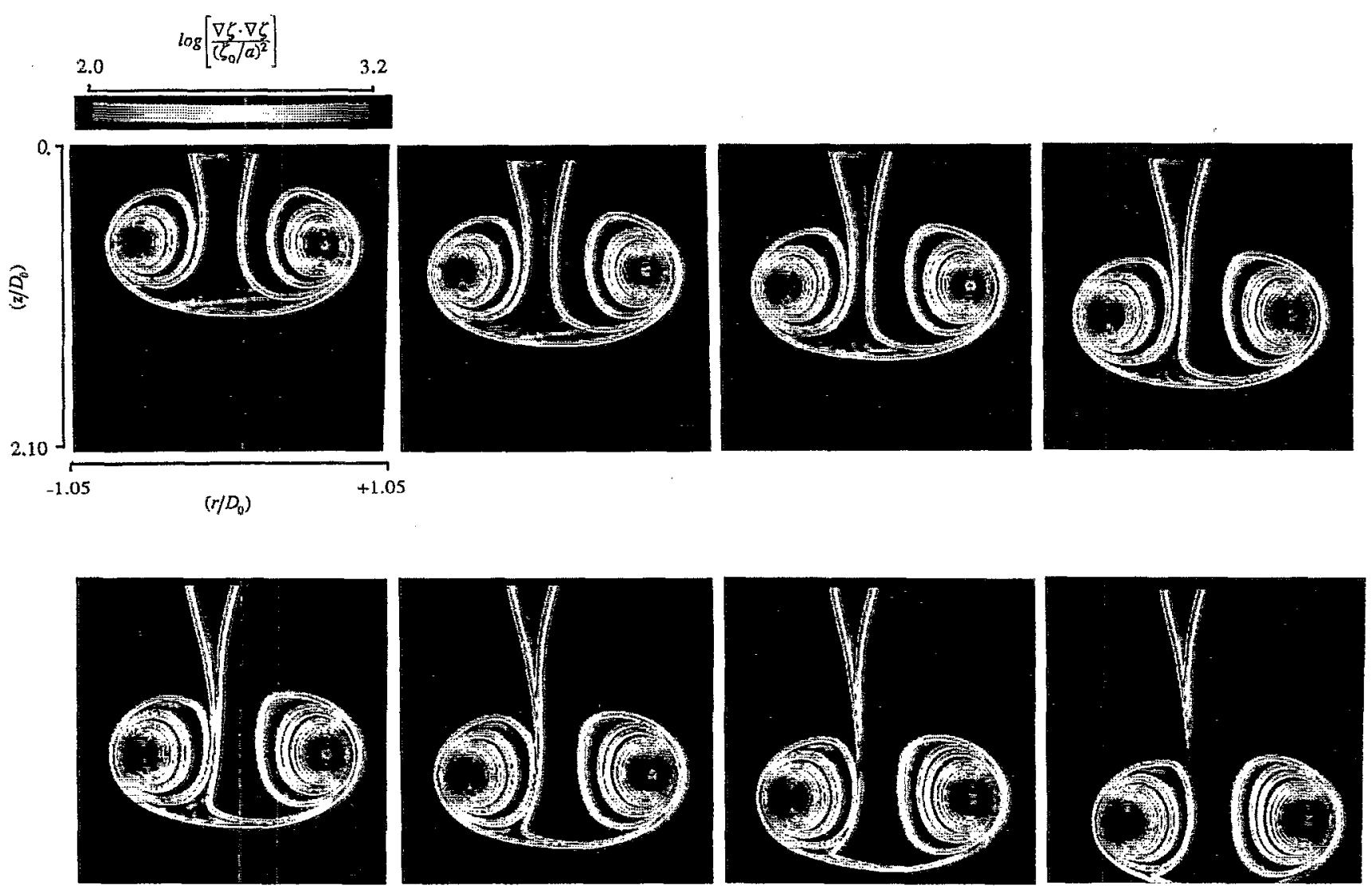

FIG. 4. The logarithm of the mixing rate field $\log \nabla \zeta \cdot \nabla \zeta(\mathrm{x}, t)$ during the pinch-off phase of the ring. The dimensionless time between successive panels shown is $\left(\Delta t \cdot \Gamma / 2 \pi a^{2}\right) \approx 0.089$. 


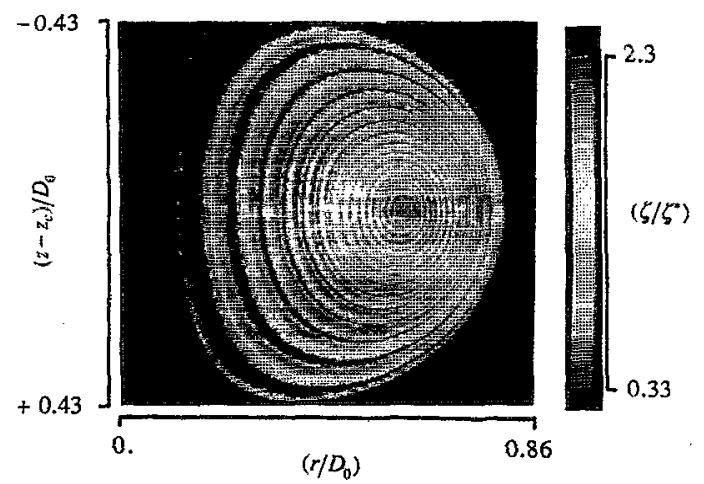

(a)

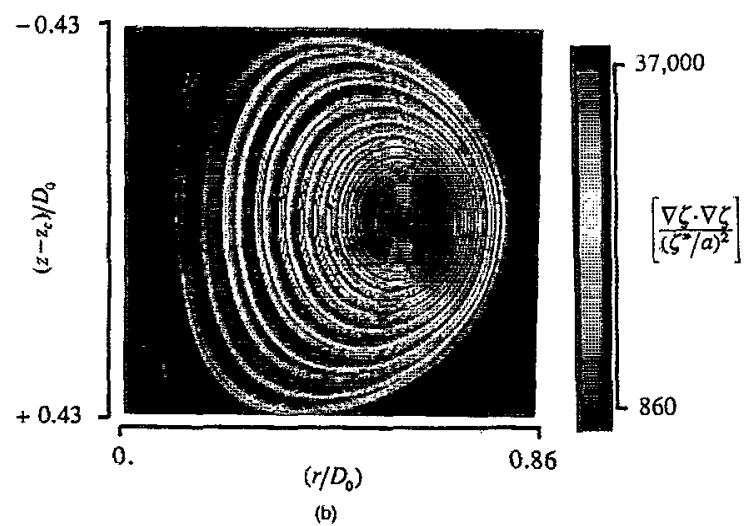

FIG. 5. The asymptotic structure of the laminar vortex ring. (a) The scalar field $\zeta(\mathrm{x}, t)$. (b) The molecular mixing rate field $\nabla \xi \cdot \nabla \zeta(\mathrm{x}, t)$.

begins where the dissipation layer extending from the forward stagnation point meets the remnants of the layers that originally were connected to the nozzle lip. This cancellation of the mixing rate within these layers continues along the spiral arms as these two layers wrap around each other in their approach toward the vortex core. As a consequence, the mixing rate begins to drop rapidly as the center of the core is approached. This will be seen more clearly in the discussion of the asymptotic stage given below. One last point regarding the pinch-off stage is important here. Notice that the two layers emanating from the nozzle do not remain symmetrically located around the centerline of the ring. Except at the earliest times in this stage, the layers consistently wander off the centerline, especially near the forward stagnation point. This appears to be due to the fact that this is a hyperbolic point, and as a result the flow along streamlines approaching it is sensitive to even very small displacements from the centerline. The effect of any slight initial asymmetry that causes these two layers not to pinch off precisely on this dividing streamline will therefore show a large displacement when integrated over time, even though the underlying flow field is very nearly symmetric.

\section{Asymptotic phase}

Once the ring has pinched off, the ambient fluid trapped inside the Kelvin oval wraps up with the plenum fluid on an increasingly finer scale along the two spiral arms extending toward the center of the core. This can be seen quite clearly in Figs. 5(a) and 5(b), which, respectively, show the scalar and scalar dissipation fields in the right half of the ring at a stage where the ring has already wrapped up to a rather large degree. As the axes indicate, the image covers only the right half of the ring, with the centerline coinciding roughly with the left edge of the image and the vertical axis referenced to the core location $z_{c}$. The number of windings seen now give an indication of the dimensionless "age" of the ring. Note that the color values are assigned as shown, with $\zeta$ * denoting the average scalar value of the fluid inside the Kelvin oval, determined without radial weighting in the plane.

The primary dynamical feature during this stage of the ring evolution is the continual lengthening of the two spiral arms leading to the core in Fig. 5(a). This extensional straining of the scalar dissipation layers in Fig. 5(b) between these arms leads to a compressional straining along the layer normal direction. This in turn creates a steepening of the gradient scale and a corresponding increase in the gradient magnitude. Eventually the thickening effect of diffusion acts to balance the thinning due to the strain, and the result is a strain-limited molecular diffusion layer thickness. In Fig. 5 (b) this balance between diffusion and the strain field imposed by continuity appears to establish a thickening of the layers as they rotate through the upper half of the ring from the farthest outboard point back toward the centerline, and then a thinning of the layers as they rotate back through the lower half of the ring. The scalar dissipation values farthest from the centerline of the ring also appear to be consistently lower than those closest to the centerline, presumably due to diffusional cancellation between the layers. This also leads to a rapid drop in the scalar dissipation within the layers as the center of the core is approached. Indeed, the scalar values in both arms of the spiral can be seen to approach the green value attained at the center of the core. Note in particular that even the core center appears to be adequately resolved, suggesting that the scalar dissipation field is sufficiently resolved throughout the ring to allow accurate determination of the fine structure of the mixing process. There is, moreover, a pattern of alternating high and low dissipation rates encountered as the core is approached along the layer normal direction. However, it is not clear if this results from the different strain rate histories to which the two scalar dissipation layers that originally emanated from the forward stagnation point and from the nozzle were subjected, or if instead this is a remnant of the initial conditions.

\section{VELOCITY FIELD MEASUREMENTS}

Equation (1) shows the effect of the underlying velocity field on the mixing process within the ring. It is possible to use these relatively high resolution scalar field measurements to invert Eq. (1) and extract a limited type of information about the velocity field underlying the mixing process. In particular, since the velocity affects the scalar field only through the $\mathbf{u} \cdot \nabla \zeta$ term in Eq. (1), the scalar field is affected only by the velocity component $u_{\|}(\mathbf{x}, t)$ oriented along the local gradient vector orientation. [Note that the velocity $u_{\| l}(x, t)$ is physically different from the notion of an isoscalar surface velocity discussed by Gibson. ${ }^{21}$ ] Inverting the con- 


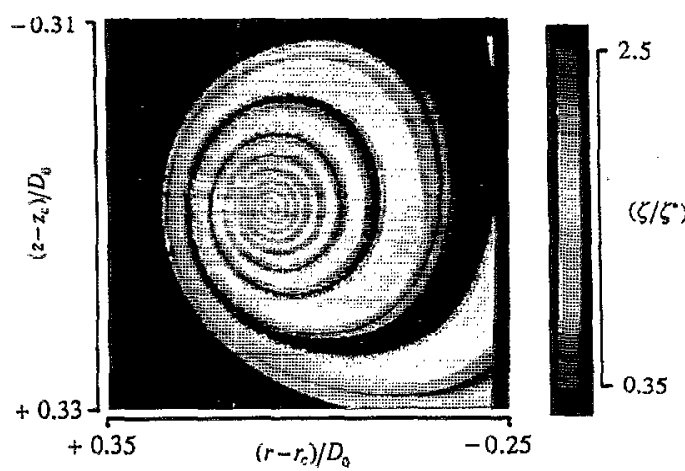

(a)

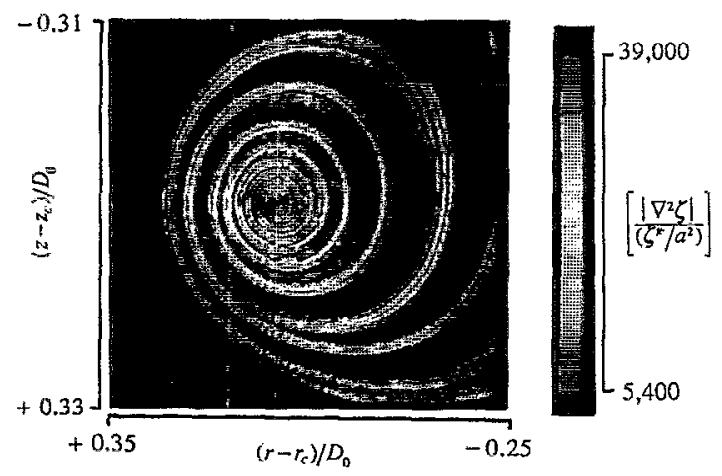

(c)

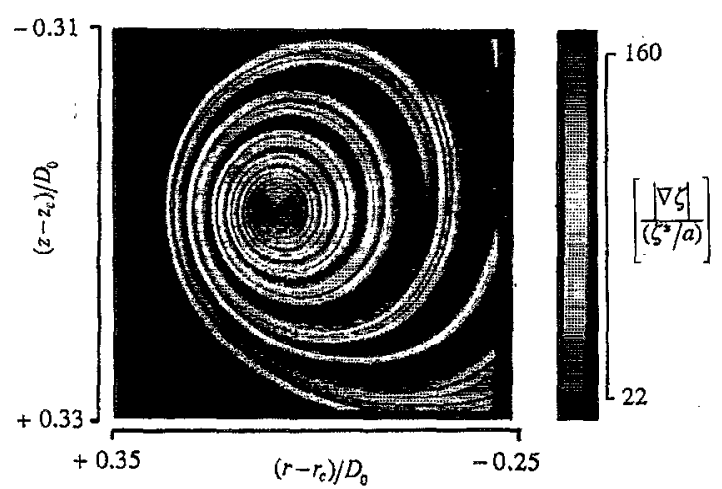

(b)

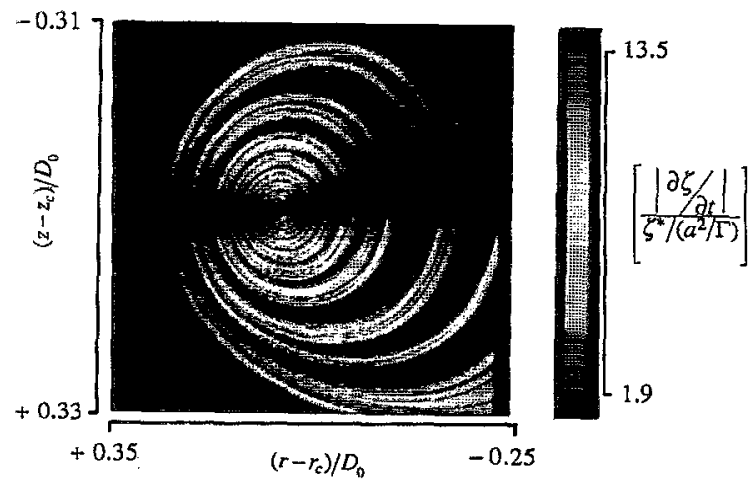

(d)

FIG. 6. The component fields involved in inverting the mixing process described by Eq. (1) to obtain the velocity field $u_{\|}$( $(x, t)$ via Eq. ( 5 ). (a) The original scalar field $\xi(\mathbf{x}, t)$. (b) The resulting gradient magnitude field $|\nabla \xi(\mathrm{x}, t)|$. (c) The resulling Laplacian field $\left|\nabla^{2} \xi(\mathbf{x}, t)\right|$. (d) The time derivative field $|\partial \xi(\mathrm{x}, t) / \partial t|$.

served scalar transport equation gives this velocity component in terms of the measured scalar field $\zeta(\mathbf{x}, t)$ as

$$
u_{\|}(\mathbf{x}, t)=\left(\frac{1}{\operatorname{ReSc}} \nabla^{2} \zeta-\frac{\partial \zeta}{\partial t}\right)(|\nabla \zeta|)^{-1}
$$

Note that this involves not only the scalar gradient field examined thus far, but also the Laplacian $\nabla^{2} \zeta(x, t)$ and the time derivative $\partial \xi / \partial t$ of the scalar field. If measurements such as these have a spatial resolution that is sufficiently high to allow accurate determination of the second derivatives in the Laplacian, and at the same time have a sufficiently fine temporal separation between successive images in time to allow meaningful evaluation of the time derivative of the scalar field, then Eq. (5) allows the velocity component $u_{\|}(\mathbf{x}, t)$ to be determined. Notice that this inversion is only possible where the scalar gradient $\nabla \xi(\mathbf{x}, t)$ is not zero.

In Fig. 6 we show each of the component fields involved in the inversion in Eq. (5). The colors in each case denote linearly increasing values of the absolute magnitude of the component field, and no explicit smoothing or filtering has been applied to any of these fields. Here $\xi *$ denotes the average scalar value in the spiral windings. Figure 6(a) shows the original scalar field $\xi(\mathrm{x}, t)$ during a relatively early stage in the pinch-off phase. The resulting scalar gradient magnitude $|\nabla \zeta(\mathbf{x}, t)|$, determined as described in Sec. III, is shown in Fig. 6(b). Numerically evaluating the divergence of this scalar gradient field, again using linear central difference approximations, yields the Laplacian field $\nabla^{2} \xi(x, t)$ shown in Fig. 6(c). Here it can be seen that the original scalar field data are sufficiently highly resolved to allow a relatively smooth determination even of these second derivatives. We note, however, that the relatively high value of $\operatorname{Re} S c$ involved in these measurements renders the contribution of the Laplacian in Eq. (5) practically negligible, and thus the requirement that second derivatives of the measured data need to be accurately obtainable can in practice be relaxed. As far as the time derivative $\partial \zeta / \partial t$ is concerned, we also approximate this with a linear central difference among the scalar field images immediately before and immediately after that shown in Fig. 6(a), with the result shown in Fig. $6(d)$.

From the component fields in Fig. 6, the inversion in Eq. (5) can be evaluated to determine $u_{\|}(\mathbf{x}, t)$. The result is shown in Fig. 7, where the colors from blue to red denote linearly increasing values of $u_{\|}$, and where points at which the scalar gradient magnitude in Fig. 5(b) is essentially zero have been flagged black to show where the inversion is not possible. Note also that the $u_{\|}(\mathrm{x}, t)$ is presented in the original lab-fixed frame. The result obtained shows relatively high values along the upstream and downstream sectors of the ring, where the scalar gradient lies coincident with the direction of propagation, and much smaller values where the gradient vector is oriented largely normal to the propagation 


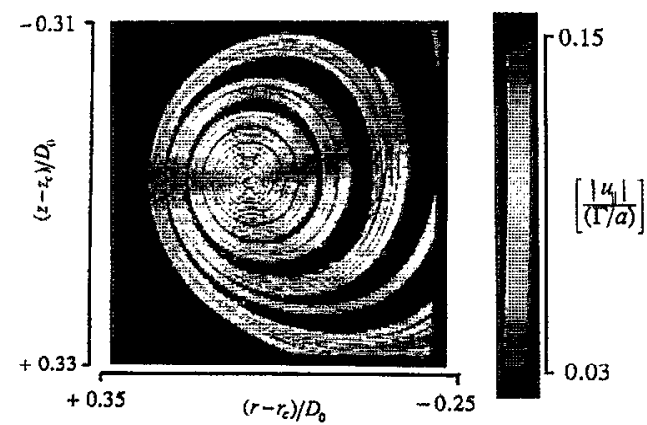

FIG. 7. The velocity component along the local scalar gradient vector direction, $\left|u_{\|}(\mathbf{x}, t)\right|$, obtained from the component fields in Fig. 6 via Eq. (5). Colors from blue through red denote linearly increasing values of $\left|u_{\|}(x, t)\right|$.

direction. Continuity can in principle be used to extract the remaining velocity component $u_{\perp}(\mathbf{x}, t)$ from the result obtained for $u_{\|}(\mathbf{x}, t)$ in Fig. 7 , since the velocity at the center of the core is known from the propagation speed of the ring. This perpendicular component, however, contains no direct information about the mixing processes in the ring, and instead results entirely from the constraint of continuity. Nevertheless, it is interesting to note that this would allow the strain rate tensor $\epsilon(\mathbf{x}, t)$ in $\mathrm{Eq}$. (3) to be evaluated, thereby permitting a quantitative study of the interaction between the strain field and the mixing that extends well beyond the relatively detailed documentation of the mixing processes in the ring undertaken here. As a practical matter, this method for obtaining the underlying velocity field from detailed measurements of molecular mixing in certain axisymmetric or two-dimensional flows has applications beyond the study of vortex rings. In particular, in appropriate three-dimensional flows the vorticity transport equation together with the requirement of continuity may provide the necessary constraints to at least in principle determine the two remaining velocity components perpendicular to $u_{\|}(\mathrm{x}, t)$ obtained from mixing measurements such as these via Eq. (5).

\section{CONCLUDING REMARKS}

The results presented here provide the first significantly detailed experimental examination of the molecular mixing processes associated with dynamically passive conserved scalar quantities in an axisymmetric laminar vortex ring. They identify three relatively distinct stages in the evolution of the mixing rate field, namely (i) the formation phase, (ii) the pinch-off phase, and (iii) the asymptotic laminar phase. In each phase, the diffusional cancellation term arising in the scalar dissipation transport equation is found to play a key role in limiting the total amount of mixing that takes place. We note also that the layer-like fine structure seen in the dissipation field in each of these three phases, together with the overall similarity in the dynamics of the dissipation field and the vorticity magnitude field, are suggestive of a layerlike fine structure in the vorticity field at relatively large Reynolds numbers. The results presented here also show that it is possible to extract information about the underlying velocity field in which the mixing takes place from such relatively highly resolved scalar field measurements. Moreover, the fact that in laminar vortex rings the velocity in the core is known suggests the use of continuity to extract the strain rate and vorticity fields from measurements such as these of the scalar mixing process. This would permit very highly detailed quantitative studies of the interaction of the strain rate and vorticity fields with the molecular mixing process documented here. In this sense, the axisymmetric laminar vortex ring provides a uniquely simple and carefully controllable flow, simultaneously accessible to experimental, numerical and theoretical study, in which the precise understanding of various aspects of the mixing processes in fluid flows can be significantly extended.

\section{ACKNOWLEDGMENT}

The work presented here is being supported, in part, by the Air Force Office of Scientific Research (AFOSR) under AFOSR Grant Nò. 89-0541.

${ }^{1}$ D. Auerbach, J. Fluid Mech. 183, 185 (1987).

${ }^{2}$ D. Auerbach, Fluid Dyn. Res. 3, 209-213 (1988).

${ }^{3}$ N. Didden, Z. Angew. Math. Phys. 30, 101 (1977).

${ }^{4}$ L. E. Fraenkel, Proc. R. Soc. London Ser. A 316, 29 (1970).

${ }^{5} \mathrm{~J}$. Z. Irdmusa and C. A. Garris, AIAA J. 25, 371 (1987).

${ }^{6}$ C. -H. Krutzsch, Z. Angew. Math. Mech. 16, 352 (1936).

${ }^{7}$ C.-H. Krutzsch, Ann. Phys. (Leipzig) 35, 497 (1939).

${ }^{8} \mathrm{~J}$. Jarusinski, J. H. S. Lee, and P. Knystautas, Proceedings of the 22nd International Symposium on Combustion (The Combustion Institute, Pittsburgh, 1988), pp. 505-514.

${ }^{9}$ P. F. Linden, J. Fluid Mech. 60, 467 (1973).

${ }^{10}$ R. H. Macgarvey, and C. S. MacLatchy, Can. J. Phys. 42, 678 (1964).

"R. H. Macgarvey, and C. S. MacLatchy, Can. J. Phys. 42, 684 (1964).

${ }^{12}$ T. Maxworthy, J. Fluid Mech. 51, 15 (1972).

${ }^{13}$ T. Maxworthy, J. Fluid Mech. 81, 465 (1977).

${ }_{14}$ P. G. Saffman, Stud. Appl. Math. 49, 371 (1970).

${ }^{15}$ P. G. Saffman, J. Fluid Mech. 84, 625 (1978).

${ }^{16} \mathrm{~S}$. E. Widnall and J. P. Sullivan, Proc. R. Soc. London Ser. A 332, 335 (1973).

${ }^{17}$ S. E. Widnall, D. B. Bliss, and C.-Y. Tsai, J. Fluid Mech. 66, 35 (1974).

${ }^{18}$ W. J. A. Dahm, C. M. Scheil, and G. Tryggvason, J. Fluid Mech. 205, 1 (1989).

${ }^{19}$ W. J. A. Dahm and K. A. Buch, Turbulent Shear Flows 7 (Springer, Berlin, 1990).

${ }^{20}$ W. J. A. Dahm, K. B. Southerland, and K. A. Buch, Phys. Fluids A 3, 1115 (1991).

${ }^{21}$ C. H. Gibson, Phys. Fluids 11, 2305 (1968). 